\title{
Pollution of Natural Ecosystems and the Biosphere Radionuklidamy and Its Implications
}

\author{
RAE Aliyev ZH* \\ Erosion and Irrigation Institute of the Azerbaijan National Academy of Sciences
}

Received: May 29, 2018; Published: June 07, 2018

*Corresponding author: RAE Aliyev ZH, Erosion and Irrigation Institute of the Azerbaijan National Academy of Sciences, Russia

\section{Abstract}

Now humanity is understood not only the magnitude of its creativity, but its complete dependence on the environment Wednesday. However, this understanding until nevseob» by and often submissive short-term selfish interests (situation, economy, etc.); ended in the twentieth century, a century of great discoveries and global achievements in the sciences, engineering, economics, and human culture.

Keywords: Pollution; ERE: Earth and Natural Radioactive Elements

Abbreviations: ERE: Earth and Natural Radioactive Elements; OCSP: Organochlorine Pesticides; FOP: Phosphorus-Organic Pesticide

\section{Introduction}

In this century, along with the progress occurred global destruction of nature, pollution Wednesday. Scientific and technical progress was threaten green civilization. From the middle of the century, mankind departed entered a new phase of its development in the noosphere i.e. when the only alternative becomes a balanced national economy conducting "joint" with all the elements of nature, i.e. on a smoke break already established natural biogeocenozam populations and communities, and combining with them, complementing them, sozhitelstvuja them.

\section{Progress in the Study and Discussion of Materials}

Powerful development of the productive forces (especially in the highly industrial relations) current forecast assumptions, insistently demands to send them in an environmentally sound manner. Maximize production and profit now becomes the criterion for the well-being of any Community (State). So now everything should be subjected to environmental assessment it is deep understanding of the current natural environment becomes the basis for further action by any community, because the scope of the activities of mankind became function the State of nature, i.e. humanity, maybe for the first time began to feel not only the scope of its activities, but also their dependence on State Wednesday.

\section{The Objectives of the Study}

Taking into account the fact that such dependence, human intelligence above all seeks to systematize the facts of anthropogenic impact and identify the most significant priorities for health and environmental protection Wednesday.

These priorities are primarily aimed at preserving human homeostasis and everything else that surrounds the person i.e. Wednesday, with which a person communicates energy. If we talk specifically about the priorities, the first thing you should pay attention to the components of the biosphere, accumulation of biogenic elements (all forms of nitrogen: $\mathrm{NH}_{4}, \mathrm{NO}_{3}, \mathrm{NO}_{2}$, as well as $\mathrm{P}_{2} \mathrm{O}_{5}$, Fe AGG . SI $\mathrm{ON}_{2}$ ), i.e. items that possess biological activity and how the rules of outlier of biological cycles of substance due to its excess akkumulirovannosti this specific biota, which ultimately leads to the formation of taksogennoj zone, i.e. the formation of abiotic Wednesday. In addition, education excess with $\mathrm{CO}_{2}$, as well as various oxides and nitrogen gidridi in the atmosphere together with various volatile hydrocarbons, are powerful toksikogenami of life in the biosphere causes necrosis Wednesday human habitation. However, if you take into account the targeted use of OCSP (An Organochlorine Pesticides) and FOP (Phosphorus-Organic Pesticide) man, it becomes apparent the brunt of toksikogennoj load obrushivajushheesja on him.

However, in the series of all these toxic substances, a special immeasurably insidious and having serious consequences, has radionuclide pollution of the natural environment. It should be said about the special role of this pollution, which contributes to carcinogenesis in living organisms. 
Thus, specific toxicants, polluting soils, water sources and the atmosphere and determining the general radio-ecological situation, are radionuclides, which are unstable isotopes of many chemical elements that spontaneously dissociate for humans with the release of radiation energy. Its action on a living organism is very specific and consists in the following.

In radioactive decay, when the rays are released, their penetration into any external environment begins, including and living organisms. By themselves, these rays, penetrating the body, are able to ionize the constituent elements of the cell (especially the rays); for example, hemoglobin of blood. At under normal conditions, the protein (hemoglobin) is electro-neutral, but when it penetrates, for example, the radiation, this hemoglobin becomes negatively charged, because a proton is embroidered from it, which means that it practically can no longer be functional in transferring oxygen from the lungs to the brain, the heart, etc., the bloodstream. Moreover, a negatively charged hemoglobin, i.e., in the free-radical state, promotes the neighboring hemoglobin to lose its proton, i.e. The ionization wave (depending on the dose of radiation) increases in an avalanche manner, which in the end can lead to an organism lethal outcome. Thus, from an ecological point of view, radioactive radiation is an extremely formidable abiotic factor.

However, it should be specially noted that in optimally low doses, radioactive radiation even acts favorably on the body, which is manifested in activation of redox reactions of the body, in optimizing the respiratory coefficient of the hormonal system, etc [1-3].

At present, it is known that ionizing radiation is composed of three sources of ionizing radiation [3]:

a) Cosmic rays reaching the surface of the Earth.

b) Radiation of radioactive elements that make up the earth's crust (soils, rocks, seawater, soil water, etc.).

c) Radioactive radiation caused by radionuclides, which are part of the organisms of humans (and also animals).

All three types of radiation form a natural radio background (EPF). The total radiative load per person in modern society is estimated to be about $2 / 3$ of the effect of ionizing radiation and $1 / 3$ of the impact of anthropogenic sources.

It should be noted that the EPF varies in different regions of the planet in the range of values that differ by more than 2 orders of magnitude. The biota of these regions has evolved to adapt to its EPF. And the danger to living organisms can only be the excess of the EPF caused by artificial radioactivity [4].

With the current state of the biosphere, when emissions, incl. and carcinogenic substances into it is carried out uncontrollably chaotically, convection and other processes can spontaneously localize dispersed radionuclides thousands of kilometers from the centers of their formation, at any point of the planet.

And such artificial radioactivity in the external environment is due to various reasons: tests of nuclear weapons; in reactor accidents Nuclear power plants and when they are cooled; at disposal of uranium ore processing waste; when using ionizing radiation in medicine (diagnostics, therapy). The last radiation load reaches about $30 \%$ of the total human ionizing radiation load from all possible sources [3].

Thus, at the present time in nature there is a natural radionuclide ionization, which causes the EPF and the artificial radionuclide contamination, which can lead to irreversible transformations in the animal body, depending on the doses of the radiation level; violate its homeostasis, evolutionarily conditioned both ontogenetic and phylogenetic. In this regard, there is a need to develop environmental criteria (they can simultaneously be sanitary and hygienic) for the main types of soils, natural waters and the air, i.e. of the environment with which the person must be in complete harmonious harmony.

Objectives of the study: is to study the characteristics of the natural environment by radionuclide contamination.

Atmosphere: As a rule, in the atmosphere, radionuclide contamination is in the aerosol state. And the convection currents due to the temperature in the barometric ingredients, in a certain moment under the influence of gravitational forces settle on soil, vegetative, aquatic and so on Surface. According to the radiation safety standards (NRB-76/87, the permissible concentration of strontium-90 radionuclide in atmospheric air should be 4.0,10-14) km/ liter, and for cesium-137 such a standard has not yet been obtained.

Hydrosphere: The main transporting and accumulating (in these sediments) environment in the "water-soil" -plants-human system. "It is in the aqueous medium that suspended radionuclides of the atmosphere dissolve: it is in this state that they penetrate the soil, underground layers of groundwater and other waters (including drinking sources).

At present there are no officially approved ecological criteria for assessing the level of radioactive contamination (SRH) of aquatic ecosystems. Again, there are only radioactive safety standards (NRB-76/87) containing information from the allowable concentration of individual radionuclides in water (DKV), for which this figure corresponds to the ratio of the annual intake limit or its a critical body during the year [1]. So the value of DKV is the most widespread and dangerous in water objects long-lived radionuclides of strontium-90 and cesium-137 is 4.0, respectively. $10-10$ $\mathrm{km} /$ liter (for bones) and 1.5-6.5 - 10-8 km/liter (for the whole body and liver). These official parameters (norms) for assessing the radioecological situation (situation) are only indicative; they are designed for sanitary purposes.

Particularly it should be said that radionuclides migrate, accumulate and transform in all major biocurrents and components of the biota of water bodies with the involvement of substances in the biotic cycle. In fresh water bodies and watercourses, most of the radionuclides are sorbed by these sediments and higher aquatic vegetation. The smaller part of the radionuclides remains more or less evenly distributed dissolved in water. This is a very important in the theoretical and practical sense of the law. The fact is that in the bottom sediments of water bodies, due to the high sorption capacity of 
the silt, so large amounts of radionuclides are usually accumulated, that the water of the water column in those water bodies can be "clean" in accordance with sanitary and hygienic criteria.

However, the radiological well-being of water bodies contaminated with radionuclides, even if they are radio-intensive, is always very relative, because:

a) Firstly, hydrosites are unable to withstand external radiation radiation at doses that deviate significantly from the optimal values;

b) Secondly, the needs in the process of the biotic cycle are able to accumulate radionuclides in such quantities (high concentrations) in the body that these accumulated radionuclides become life-threatening, as well as the health (physiological state) of their consumers (consumers), including humans.

It is also important that in the radio-contaminated water bodies in the greatest quantity there are usually two radionuclides-Strontium -90 and cesium-137, which have a long half-life and high toxicity, which are dangerous.

Once in the body of hydrobionts, radionuclides become sources of the incorporated chronic radiation, especially strong in that If they concentrate in certain tissues and form dangerous foci locally radiating nearby cells. With chronic action of even small doses of radiation, a accumulative (total) effect is produced that causes a mutagenic and damaging cell, organ, organism of action. In this case, a negative radiation effect can occur with minimal amounts of energy absorbed by the irradiated body (organism).

Chronic irradiation with radionuclides of hydrobionts of all trophic levels in ecosystems of water bodies to varying degrees of radio contamination can lead not only to the cessation of fishing due to the unfitness of fish for human and animal nutrition, but also to other unforeseen consequences.

Possible disappearances of some species and an outbreak of the number of other fish species that have acquired favorable mutations for them. This leads to a restructuring of the biota structure and changes in biotic links, which can worsen the hydrobiological processes of water quality and productivity in water bodies.

The Pedosphere: The natural radioactive background (EFR) of the soil is determined by such elements as Ra, Ac, Gh. K-40, which undergo spontaneous successive transformations, forming families of radioactive elements and isotopes with very different half-lives. To a lesser extent, 0-14 are present in the soil. A high indicator is fixed in some ore deposits; a small indicator of EPF is fixed almost on the whole surface of the Earth and Natural Radioactive Elements (ERE) are present in all rocks, soils and waters.

The concentration of ERE in soil depends on their content in the parent (rock) rock and the degree of weathering of it as a result of soil formation. In soils formed on the weathering products of acidic rocks, radioactive elements and isotopes is contained more than in soils formed on basic or ultrabasic rocks. Soils of heavy mechanical composition contain ERE more than the soil of the lung mechanical composition. Migration of ERS depends largely on the landscape: the soil of eluvial and trans-eluvial landscapes contain significantly more of Gh and less than Ra; in the soils of accumulative landscapes, the content of $\mathrm{R}$ increases with respect to the average. In soils, in the soils, C-1.10-4, Gh-6.10-4, Ra-B.10-4, potassium-40 -2.10- 4.

The artificial radioactive background of the soil is mainly determined by atomic and thermonuclear explosions or wastes from the nuclear industry. As a result, radioactive elements and isotopes that do not occur in the EPF appear in the soil. The long-lived radioactive elements fall into the soil, including Cp-30, Cs-137, Ru-106, L-144, $\mathrm{Mn}-54$, etc. The most important values are $\mathrm{Cp}-90$ and $\mathrm{Cs}-137$. The intensive is sorbed by the soils in the exchange state, absorbed by the root systems and through through the plant are included in the agricultural links of migration of elements of mineral nutrition. On some soils containing minerals like vermiculite.

Cs-137 is readily available to plants, Cp-90 and Cs -137 represent the greatest danger because they are close analogues of physiologically important elements-Ca and $\mathrm{K}$, have a long half-life and high energy of radiation (Cp-90-emitter, Cs-137 - and-emitter), can easily be incorporated into the biological cycle and enter human organisms. The content and distribution of Cp-90 and Cs - 137 in soils is determined by the intensity and character of their loss from the atmosphere, the properties of the isotopes and soils themselves, and, on the whole, by the aggregate of all natural conditions (vegetation, climate, relief).

The distribution of the precipitating Cp-90 and Cs -137 over the territory depends on the latitude of the terrain. The maximum loss is observed within $30-500 \mathrm{C}$ latitude.

The fixing and content of Cp-90 and Cs -137 in soil depends on the form in which compounds they are (water-soluble base-exchange, hardly soluble), and also on soil properties.

When cultivating agricultural crops on soils contaminated with IPE, the consequence is extracted by plant roots and accumulated in plant products. Reduce their intake in plants can be appropriate methods. Studies have shown that $\mathrm{Cp}$-90 in comparison with Cs -137 intensively enters the plants, which is explained by the different degree of their fixation in the soil. Calcium-loving plants usually absorb relatively more than $\mathrm{Cp}-90$, than the poorest plants most accumulate Cp-90, legumes, less-root crops and even less - elastics. Plants containing more potassium absorb and more Cs-137. On soils of light mechanical composition and poor humus, other things being equal, radioactive elements enter the plants more than on soils of heavy mechanical composition and rich in humus. The intake of Sr-90, in plants decreases on cultivated soils with $\mathrm{m} 3$ sequestration and fertilization. The intake of Cs-137 in plants reduces various potassium fertilizers, the intake of Cs-137 is inhibited by the introduction of alkali metal phosphates.

\section{Ionizing Radiation is a Powerful Mutagenic Factor}

Taking into account all planetary prevalence of radioactive contamination and all the harmfulness of its presence in biota, it becomes extremely necessary to examine its impact on the most 
important genetic Fund of mankind, i.e. becomes acutely necessary to predict how can education the ionosphere (the scope of the human mind) that naturally due to the planet's gene pool.

After all, under the influence of small doses of radiation mutation frequency increases dramatically. The vast majority of mutations generates various genetic deformities and diseases. Nakap-livajas in generations, they can bring great suffering to humankind. And the duty of a modern society is not only to save the life of the current living generations, but also to protect future generations from the ancestral burdens harmful mutations.

Submission Link: https://biomedres.us/submit-manuscript.php

This work is licensed under Creative Commons Attribution 4.0 License

\section{References}

1. Romanenko VD, Oksijuk OP, Zhukinskij VN, A Stolberg (1990) Environmental assessment of the activity of hydrotechnical building on water objects. Naukova dumka, Kiev.

2. Lavrik VI (1984) Human Heredity and the environment Wednesday. Soviet Committee for the UNESCO programme «man and the Biosphere Programme, Nauka». Moscow, USA.

3. NP Napalkova (1989) Academy of Medical Sciences of USSR «medicine». General Oncology.

4. (1987) Kuzin-problems of modern radiobiology.

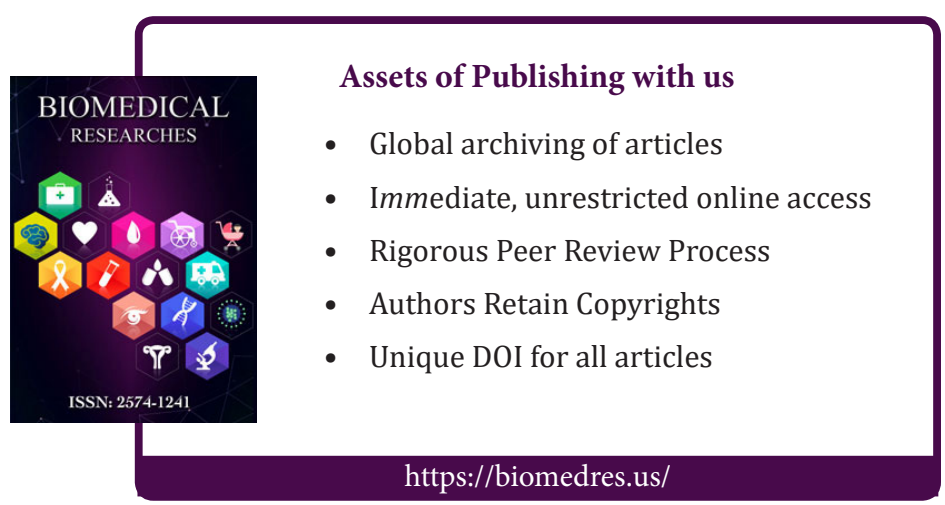

